\title{
COMBO-17+4: An Optical-NIR Survey for Galaxies out to $\mathrm{z}=2$
}

\author{
M-H. Nicol ${ }^{1}$, K. Meisenheimer ${ }^{1}$, C. Tapken ${ }^{1}$ and C. Wolf ${ }^{2}$ \\ ${ }^{1}$ Max-Planck Institut für Astronomie, Königstuhl 17, 69117 Heidelberg, Germany \\ email:nicol@mpia-hd.mpg.de \\ ${ }^{2}$ Departement of Physics, Denys Wilkinson Bldg.,University of Oxford, \\ Keble Road, Oxford, OX1 3RH, UK
}

\begin{abstract}
Classifying Object by Medium-Band Observations in 17 filters (COMBO-17) has already produced a very accurate picture of galaxy evolution since $\mathrm{z} \sim 1$ based on 25000 galaxies in 17 medium optical bands. We now extend the range of reliable multi-color redshifts with COMBO-17+4, a deep optical-NIR survey which will combine the existing optical data set of COMBO-17 with near infrared observation in three medium bands : $\mathrm{Y}(\lambda / \Delta \lambda=1040 / 80 \mathrm{~nm})$, $\mathrm{J} 1(1190 / 130 \mathrm{~nm})$ and J2(1320/130nm) and one broad band H(1650/300nm). The NIR bands extend the photometric redshift range to $\mathrm{z} \sim 2.1$. COMBO $17+4$ will provide the first large sample of galaxies $(>5000)$ at $1<\mathrm{z}<2$ with a redshifts accuracy of $\Delta \mathrm{z}<0.03(1+\mathrm{z})$. Three fields are observed: Abell 901, Abell 226 and the COMBO 11h-field, for a total coverage of $0.77 \square^{2}$ of the sky. Each COMBO 17+4 field measures $31 \times 30$ sqarcmin. The NIR bands are observed with the Omega2000 camera at Calar Alto Observatory in Spain.

The scientific goals for this study are multiple. COMBO- $17+4$ will enable us to establish the luminosity function for the red sequence and blue galaxies in the redshift range $1<\mathrm{z}<2$. Also it will be possible to determine the formation history at $\mathrm{z}=2$ by analyzing the width of the red sequence galaxies. Moreover this survey will provide several thousand of individual galaxy masses (with an accuracy <30\%) obtained with Spectral Energy Distribution (SED) template fitting. Once the masses are obtained the mass function will provide a useful tool to test the hierarchical model of evolution of galaxies by checking whether the massive red sequence galaxies $(\log \mathrm{M}>10.5)$ are already in place at $\mathrm{z}>1.5(9 \mathrm{Gyr})$.

We present first results from the full 21 bands photometry in half of the Abell 901 field. It allows us to study not only $\mathrm{z}>1$ galaxies but also the stellar content of several hundred cluster galaxies.
\end{abstract}

Keywords. surveys, galaxies: evolution, galaxies: photometry.

\section{References}

Bell, E F., Wolf, C., Meisenheimer, K., Rix, H-W., Borch, A., Dye, S., Kleinheinrich, M., Wisotzki, L. \& McIntosh, D H. 2004, ApJ 608, 752

Wolf, C., Meisenheimer, K., Rix, H.-W., Borch, A., Dye, S. \& Kleinheinrich, M. 2003, A\&A 401, 73

Wolf, C., Meisenheimer, K. \& Röser, H.-J. 2001, A\&A 365, 660

Wolf, C., Meisenheimer, K., Röser, H.-J., Beckwith, S. V. W., Chaffee, F. H. Jr., Fried, J., Hippelein, H., Huang, J.-S., Kümmel, M., von Kuhlmann, B., and 5 coauthors 2001, A $\varepsilon A$ 365, 681 